\title{
Abundances for metal-poor stars with accurate parallaxes ${ }^{\star}$
}

\section{II. $\alpha$-elements in the halo}

\author{
R. G. Gratton ${ }^{1}$, E. Carretta ${ }^{1}$, S. Desidera ${ }^{1}$, S. Lucatello ${ }^{1,2}$, P. Mazzei ${ }^{1}$, and M. Barbieri ${ }^{3}$ \\ 1 INAF-Osservatorio Astronomico di Padova, Vicolo dell'Osservatorio 5, 35122 Padova, Italy \\ 2 Dipartimento di Astronomia, Università di Padova, Italy \\ 3 CISAS, Universita' di Padova, Italy
}

Received 17 March 2003 / Accepted 13 May 2003

\begin{abstract}
Abundances for $\alpha$-elements and Fe in about 150 field subdwarfs and early subgiants with accurate parallaxes and kinematic data are used to discuss the run of abundance ratios in metal-poor stars in the solar neighborhood. Based on kinematics, we separated stars into two populations: the first one has a positive velocity of rotation around the galactic center, and it is likely to be related to the dissipational collapse of the galaxy; the second one has either negligible or negative rotational velocity, and it is likely related to an accretion component. The two populations show a large overlap in metallicity. However, they show distinct chemical properties. For the first population we found that there are close correlations (with small scatters around) of the rotational velocity with metallicity and with the Fe/ $\alpha$ abundance ratio: this might be a signature of a not very fast collapse of the progenitor clouds, with enough time for a significant contribution by SNe Ia, although this result needs to be confirmed by a 3-D/non-LTE study. On the other side, the second population exhibits a larger scatter in both the above mentioned relations, and on average, a larger $\mathrm{Fe} / \alpha$ ratio at a given metallicity, suggesting a larger scatter in ages. We argue that the lack of stars with moderate rotational velocities and high $\mathrm{Fe} / \alpha$ abundance ratios is due to the short merging time for protogalactic clouds with prograde motion, while the presence of a group of counter-rotating stars with this characteristics indicates a much longer typical lifetimes for protogalactic fragments having such a motion. Finally, we found that perigalactic distances correlate with the $\mathrm{Fe} / \alpha$ abundance ratios better than the apogalactic distances.
\end{abstract}

Key words. stars: abundances - stars: evolution - stars: Population II - Galaxy: globular clusters: general

\section{Introduction}

Galaxy formation theory must account for the wide diversity of observed structures, from dwarf irregulars to grand design spirals, from low brightness dwarfs to luminous ellipticals. This involves at least two critical ingredients: the formation of dark halos and global formation of stars. The hierarchical clustering is currently the most successful paradigm of structures formation. In the past two decades numerical simulations have become an important tool for exploring the detailed predictions of these models (Weinberg et al. 2002). However, extending these studies to understand the formation of luminous components of galaxies is difficult since our knowledge of the star formation process and its interaction (feedback) with the surrounding interstellar medium is still rather limited (Kay et al. 2002). Local Group galaxies, and in particular our own

Send offprint requests to: R. G. Gratton, e-mail: gratton@pd.astro.it

* Based in part on data collected at the European Southern Observatory, Chile, at the MacDonald Observatory, Texas, USA, and at the Telescopio Nazionale Galileo, Canary Island, INAF, Italy-Spain.
Galaxy, represent a unique opportunity to tune these models. Recently, given the improved capabilities of new generation computers, which allow us to carry out very high particle resolution runs, the first numerical simulations of disk dominated galaxy formation in a fully consistent cosmological framework have been performed (Samland \& Gerhard 2003; Abadi et al. 2002; Governato et al. 2002). There are, however, many unresolved problems affecting both dark matter and galaxies in such a framework (Steinmez 2001; Silk \& Bouwens 2001). Detailed studies of the Galaxy can help our understanding of the complex processes involved in baryons dissipation (see e.g. the review by Freeman \& Bland-Hawthorn 2002). Eggen et al. (1962) were the first to show that it is possible to study Galaxy evolution using stellar abundances and stellar dynamics. They derive that metal-poor stars are in the halo that was created during the rapid collapse of a relatively uniform, isolated protogalactic cloud shortly after it decoupled from the universal expansion. In this scenario, gradients of metallicity and age with respect to kinematics, as represented by rotational velocity or eccentricity, should be present (see e.g. Larson 1974, 1976), and the morphology of a galaxy should be related to the 
activity of star formation in early epochs compared to the collapse time. Angular momentum conservation causes the evolution of the residual gas from an original roughly spherical distribution toward a disk. This picture was challenged by Searle $\&$ Zinn (1978). They found that the galactic globular clusters have a wide range of metal abundances independent of their radius from the galactic center. Their findings pointed out that individual protogalactic clouds might have had a significant independent chemical and dynamical evolution before or during their merging into the main galactic body. Relics of this independent evolution may be traced by careful and extensive studies of chemical and dynamical properties of the earlier galactic stellar generations. The ideas of galaxy formation via hierarchical aggregation/merging of smaller units from the early Universe well agrees with the Searle and Zinn scenario of the galactic halo formation. However within this general framework, the role of the Milky Way is still to be adequately clarified. The Milky Way is a spiral galaxy located in a region of relatively low overdensity, at the edges of the Local Supercluster, with a quite significant bulge, a rather extended thin disk, a thick disk (Gilmore et al. 1989) and a halo. The presence of a dominant stellar (and gaseous) disk suggests that dissipational collapse, the main mechanism in the formation of spiral systems, had played a very important role. Moreover there are several observational facts that do not fit well in a monolithic dissipational collapse scheme. First, stars in the disk do not show the expected run of increasing metallicity with increasing age; also there is a lack of metal-poor stars (the so-called G-dwarf problem). Both these unexpected features can be understood by assuming that there is a continuous infall of metal-poor material onto the disk. The origin of this material is not clear (although a number of interstellar clouds at high latitudes have been observed since a long time, starting with IRAS satellite). Second, the real differences between the different components of our Galaxy are still to be properly understood. Gratton et al. (1996, 2000) and Fuhrmann (1998) have shown that the ratio between $\alpha$-elements (almost exclusively produced by short living core collapse Supernovae - SNe hereinafter) and Fe (a rather large fraction of which is produced on much longer time scale by thermonuclear $\mathrm{SNe}$ ) indicates a clear distinction between the epoch of formation of the thin and the thick disk, with a phase of low star formation in between. This has led several authors to construct two-infall models, that reproduce quite well these observed features (see e.g. Chiappini et al. 1997). However, the reason for this hiatus in the star formation is still unclear. It may be due to either a merger event in the early history of our Galaxy, perhaps related to the formation of the bulge of our Galaxy, or the result of a galactic wind caused by a large number of SNe explosions in the later phases of the thick disk formation (or perhaps, to both these mechanisms).

On the other side, the thick disk seems to have properties that distinguish it from the halo too: in particular, it seems much more chemically homogeneous (see Nissen \& Schuster 1997). It should be noted that the halo itself is often considered to be composed of two distinct populations, one with some rotation, and the other either not-rotating at all, or even counter-rotating (Norris 1994). The second of these populations probably traces accretion events that occurred later in the galactic history; we are even actually observing one of these merging fragment, the Sagittarius dwarf Galaxy (Ibata et al. 1995). The relation between the rotating component of the halo and the thick disk is unclear, it may well actually be a single population with a continuum of properties.

In this paper we present a discussion of the abundances of $\alpha$-elements and Fe for a group of 150 metal-poor stars with accurate Hipparcos parallaxes, whose analysis was presented in a previous paper (Gratton et al. 2003, hereinafter Paper I). High precision abundances and kinematics were obtained for these stars: this allows us to discuss some properties of the halo. In particular, we do not find any discontinuity between the properties of the rotating component of the halo and the thick disk. They agree fairly well with predictions for a dissipational collapse of a large fraction of the primordial galaxy. On the other side, the not rotating component of the halo has clearly distinct chemical properties, in agreement with scenarios where it results from accretion of protogalactic fragments with independent star formation history and chemical evolution.

The paper is organized as follows: in Sect. 2 we recall the most relevant points of Paper I (sample selection and derivation of kinematical data and chemical composition); in Sect. 3 we present the abundances, while in Sect. 4 we correlate these results with kinematical data and recall the most important biases present in our sample. Finally, in Sect. 5 we discuss our results in the framework of the early evolution of our Galaxy and present our conclusions.

\section{Sample and analysis}

Details about the analysis are described in Paper I. Briefly, we considered a sample of 146 mostly metal-poor stars with accurate parallaxes $\pi(\Delta \pi / \pi<0.2)$ from the Hipparcos catalogue (Perryman et al. 1997); accurate space velocities could be derived for these stars, combining Hipparcos proper motions and distances with radial velocities from the literature. To keep the analysis as homogeneous as possible, we did not consider stars evolved off the base of the subgiant branch. High precision equivalent widths (with errors smaller than $3 \mathrm{~m} \AA$ ) from high $S / N$, high resolution spectra were available for these stars, either from our own observations (using UVES at VLT, SARG at TNG, or the $2.7 \mathrm{~m} \mathrm{McDonald}$ telescope), or from the literature (Nissen \& Schuster 1997; Fulbright 2000; Prochaska et al. 2000).

The stars were subdivided into three groups according to kinematic criteria based on the results of appropriate Galactic orbit calculations. The groups considered are:

1. A rotating inner population, with a galactic rotation velocity larger than $40 \mathrm{~km} \mathrm{~s}^{-1}$ and $R_{\max }<15 \mathrm{kpc}$, where $R_{\max }$ is the apogalactic distance. This population includes part of what usually is called the Halo as well as the Thick Disk, and can be substantially identified with the dissipative collapse population of Norris (1994), and with the population first identified by Eggen et al. (1962). Hereinafter, we will refer to this population as Dissipative Collapse Component. The reason for our choice is that we are not be able to find 
in our data any clear discontinuity between the properties of the rotating part of the Halo and those of the Thick Disk.

2. A second population composed of non-rotating and counter-rotating stars. This population includes the remaining part of what is usually called the Halo, and can be substantially identified with the population due to accretion processes first proposed by Searle \& Zinn (1978). As we will discuss in the next sections, this population (as a group) has a chemical composition clearly distinct from that of the first one, likely due to a different origin. We will call these Accretion Component Stars.

3. Finally the Thin Disk, which also has clearly distinct chemical composition from the first population, as first showed by Gratton et al. (1996, 2000), and Fuhrmann (1998). The discontinuity in chemical composition between the Dissipative Component and the Thin Disk is likely due to a phase of low star formation that occurred during the early evolution of our Galaxy (see also Chiappini et al. 1997). For such a component:

$$
\sqrt{Z_{\max }^{2}+4 e^{2}}<0.35
$$

where $Z_{\max }$ is the maximum height above the galactic plane (in kpc) and $e$ the eccentricity of the galactic orbit of the star. With respect to the definitions of Paper I, we dropped the lower limit to $[\mathrm{Fe} / \mathrm{H}]$ for the thin disk population. In this way we have a pure kinematical definition of the various populations. Two stars were attributed to the thin disk rather than to the thick disk by dropping this criterion: HD $116316([\mathrm{Fe} / \mathrm{H}]=-0.71,[\alpha / \mathrm{Fe}]=0.19)$ and $\mathrm{HD} 134169(([\mathrm{Fe} / \mathrm{H}]=-0.84,[\alpha / \mathrm{Fe}]=0.28)$. While the impact of this change on the discussion is modest, we emphasize that stellar populations are defined on the basis of statistical criteria, and membership of individual stars may be questioned.

A part from model uncertainties (which mainly affect absolute values, but only marginally the relative ranking), the largest source of errors in our kinematic is the error in the transverse motion related to the parallax. The mean quadratic error in the parallaxes of the stars of our sample is of $\Delta \pi / \pi=0.095$. Since the average transverse motion is of $141 \mathrm{~km} \mathrm{~s}^{-1}$, the typical error in the transverse motion is of about $14 \mathrm{~km} \mathrm{~s}^{-1}$. This is much larger than the typical error in radial velocities, that for the stars in our sample is a few $\mathrm{km} \mathrm{s}^{-1}$. In order to estimate errors in the kinematical parameters, we then set up the following Monte Carlo procedure. For each star we randomly extracted a series of values for the observational parameters (parallaxes, proper motions and radial velocities) obtained from Gaussian distributions centered on the observed values and with dispersions equal to the errors assumed for these quantities. Errors in the parallaxes are those from the Hipparcos catalogue; for the remaining quantities we assumed typical values of 1 mas/yr for the proper motions and $2 \mathrm{~km} \mathrm{~s}^{-1}$ for the radial velocities. With this new set of data, we run our orbit extraction parameters. We repeated this procedure 100 times for each star, and adopted as errors in the orbital parameters the standard deviation of the distribution of the values obtained from each extraction. With this procedure we obtain that typical observational errors on the kinematic parameters we consider in this paper are $\pm 0.39 \mathrm{kpc}$ in the perigalactic distance $R_{\min }, \pm 0.028$ dex in the logarithm of the apogalactic distance $\log R_{\max }, \pm 0.046$ in the eccentricity $e$, and $\pm 12 \mathrm{~km} \mathrm{~s}^{-1}$ in the rotational velocity $V_{\text {rot }}$. Maximum errors (that is, errors for the star which is most sensitive to possible uncertainties in the observational parameters) are about twice as large. Such errors are small enough to have a negligible impact on our discussion.

A quite standard abundance analysis was carried out using these data; however, we carefully kept the star-to-star errors as small as possible. Effective temperatures for the program stars were obtained using $B-V$ and $b-y$ colors (generally assuming that interstellar reddening is negligible); these were calibrated against effective temperature using the $\mathrm{H} \alpha$ profiles that were available for about a third of the stars in the sample. A comparison with temperatures derived using the Infrared Flux Method by Alonso et al. (1996) shows an excellent agreement, with only a few slightly deviating point, generally due to stars in binary systems. The final adopted temperatures should have typical errors of about $50 \mathrm{~K}$. Surface gravities were obtained from the location of the stars in the color-magnitude diagrams, and should have errors of about \pm 0.1 dex. Finally, microturbulent velocities were obtained minimizing any trend of derived abundances from expected equivalent widths for Fe I, and have errors of about $\pm 0.3 \mathrm{~km} \mathrm{~s}^{-1}$. Accurate, updated line parameters were considered: in particular, collisional damping was considered using the recent treatment by Barklem et al. (2000), that should be much superior to the classical method based on enhancement factors over the classical van der Waals broadening given by the Unsöld formula. Moreover, oscillator strengths for $\mathrm{Mg}$ were carefully revised, and non-LTE corrections were taken into account for $\mathrm{O}$ and $\mathrm{Na}$ lines. On the other side, we do not find any evidence for significant departures from LTE for the Fe lines used in our analysis.

Finally, two features must be mentioned. First, our analysis uses standard plane-parallel atmosphere models by Kurucz (1994, with the overshooting option switched off). We found a number of small but significant discrepancies in our results that can be ascribed to limitations in these model atmospheres; such limitations can perhaps be overcome using 3-d atmosphere models, with a much more realistic treatment of convection, such as those recently used by Nissen et al. (2002). Second, a quite significant fraction of the program stars are members of binary systems. Analysis for such stars is less accurate than for single stars: in particular, significant errors can be present in the adopted effective temperatures. As evidenced in Nissen et al. (2002) and in Paper I, both these concerns are important in the analysis of some key elements, like oxygen. However, the impact on the elements considered in this paper is much smaller: for instance, we did not find any significant difference between the $[\mathrm{Fe} / \alpha]$ ratios for bona fide single stars and for stars known to be members of binary systems (at variance from what we found for the $[\mathrm{O} / \mathrm{Fe}]$ ratio).

\section{The $\mathrm{Fe} / \alpha$ ratio, and kinematics}

In Paper I we considered abundances of several $\alpha$-elements. In the present discussion we will only consider four of 

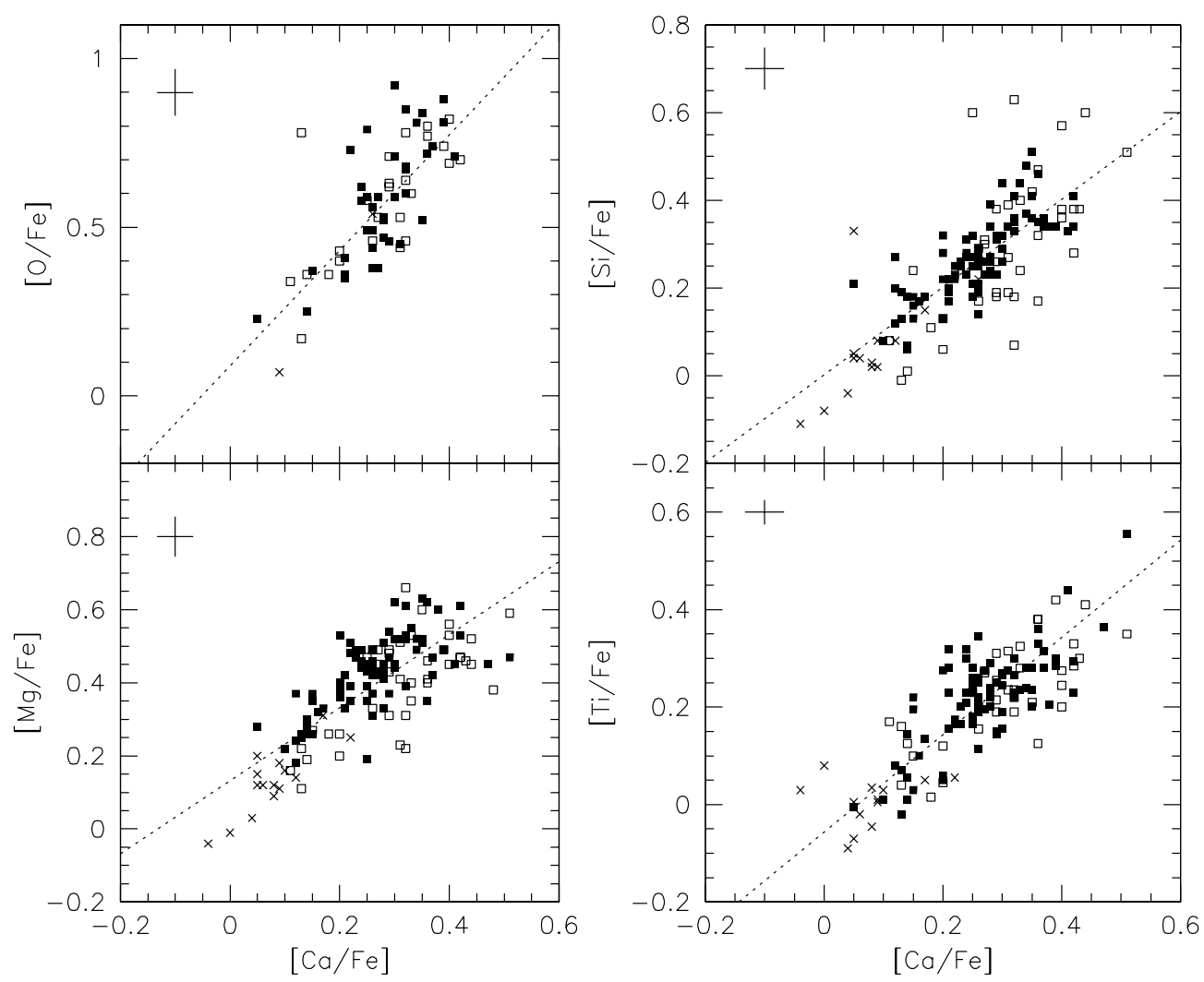

Fig. 1. Comparison between the overabundances with respect to Fe for the individual $\alpha$-elements with those obtained for Ca. Filled squares are data for Dissipative Component stars; open square those for the Accretion Component stars; crosses are data for Thin Disk stars. Error bars are shown in the upper left corner of each panel.

these elements: $\mathrm{Mg}, \mathrm{Si}, \mathrm{Ca}$, and Ti. The most abundant among $\alpha$-elements, $\mathrm{O}$, is not included because its analysis is more difficult for a variety of reasons (only the IR triplet at $7771-74 \AA$ could be used for the program stars; derived abundances are very sensitive to errors in temperatures; they are affected by significant departures from LTE; and finally they are quite sensitive to the adopted model atmosphere, with significant changes in case plane-parallel atmospheres are replaced by 3 -d ones). We simply averaged the abundance ratios to $\mathrm{Fe}$ given by the different individual elements (Ti abundances being actually the average of abundances given by neutral and singly ionized lines). Figure 1 shows the correlation existing between overabundances (with respect to $\mathrm{Fe}$ ) of the various $\alpha$-elements, using $\mathrm{Ca}$ as reference element: we selected $\mathrm{Ca}$ for this exercise because the $[\mathrm{Ca} / \mathrm{Fe}]$ is the abundance ratio most accurately determined from our data. Different symbols are used for stars belonging to different populations. Error bars for abundances of individual elements were determined in Paper I (see Table 9 of that paper). For those elements that will be considered throughout this paper, they are $\pm 0.05 \mathrm{dex}$ for $[\mathrm{Fe} / \mathrm{H}]$ and $[\alpha / \mathrm{H}]$; $0.025 \mathrm{dex}$ for the $[\mathrm{Fe} / \alpha]$ ratio (hereinafter $\alpha$ represents the average of $\mathrm{Mg}, \mathrm{Si}, \mathrm{Ca}$, and $\mathrm{Ti}$ abundances); and $0.069 \mathrm{dex}$ for the $[\mathrm{O} / \mathrm{Fe}], 0.055 \mathrm{dex}$ for the $[\mathrm{Mg} / \mathrm{Fe}], 0.048 \mathrm{dex}$ for the $[\mathrm{Si} / \mathrm{Fe}]$, $0.033 \mathrm{dex}$ for the $[\mathrm{Ca} / \mathrm{Fe}]$, and $0.030 \mathrm{dex}$ for the $[\mathrm{Ti} / \mathrm{Fe}]$ abundance ratios.

A 45 degree line is a good match to the observed data in all panels of Fig. 1, save for O. In this case, the slope is clearly larger, as expected since virtually no $\mathrm{O}$ is expected to be produced by type Ia SNe. The best fit line based on 66 stars (all but $\mathrm{CD}-80328$, that appears to be discrepant) is $[\mathrm{O} / \mathrm{Fe}]=(1.72 \pm 0.18)[\mathrm{Ca} / \mathrm{Fe}]+(0.089 \pm 0.118)$, where the error in the intercept represents the scatter of individual points. However, as mentioned in Paper I, $\mathrm{O}$ abundances are more uncertain for binary or reddened stars. Once these are eliminated, the best fit line is $[\mathrm{O} / \mathrm{Fe}]=(1.80 \pm 0.23)[\mathrm{Ca} / \mathrm{Fe}]+(0.046 \pm$ $0.100)$. Offsets are present for the remaining elements. On average, the abundance ratio to $\mathrm{Ca}$ are $0.132 \pm 0.008 \mathrm{dex}$ (rms of $0.090 \mathrm{dex}$ ) for $\mathrm{Mg}, 0.002 \pm 0.007 \mathrm{dex}$ (rms of $0.086 \mathrm{dex}$ ) for $\mathrm{Si}$, and $-0.057 \pm 0.006 \mathrm{dex}(\mathrm{rms}$ of $0.074 \mathrm{dex}$ ) for $\mathrm{Ti}$, with no significant difference between stars belonging to the dissipative or accretion components. Some of these systematic differences might be due to offsets in the analysis; however, the systematic decrease of the ratio with atomic weight corresponds well to the expected relative yields for different classes of $\mathrm{SNe}$, in particular to a systematic increase of the contribution by thermonuclear $\mathrm{SNe}$ with increasing atomic weight among the $\alpha$-elements; or to a systematic increase of the yields for the lighter elements by core-collapse SNe with increasing mass. While rather small, the rms scatter around the mean relations are larger than predicted from the errors listed in Table 9 of Paper I: note that what is relevant here are the errors in the abundance ratios to $\mathrm{Ca}$, that are expected to be $0.078,0.055,0.051$, and $0.043 \mathrm{dex}$ respectively for $\mathrm{O}$, $\mathrm{Mg}, \mathrm{Si}$, and Ti. The rms scatters remain higher even if a few (two to five) outliers are eliminated: in this case they are of $0.100 \mathrm{dex}$ for $[\mathrm{O} / \mathrm{Ca}], 0.085 \mathrm{dex}$ for $[\mathrm{Mg} / \mathrm{Ca}]$, and $0.067 \mathrm{dex}$ for 


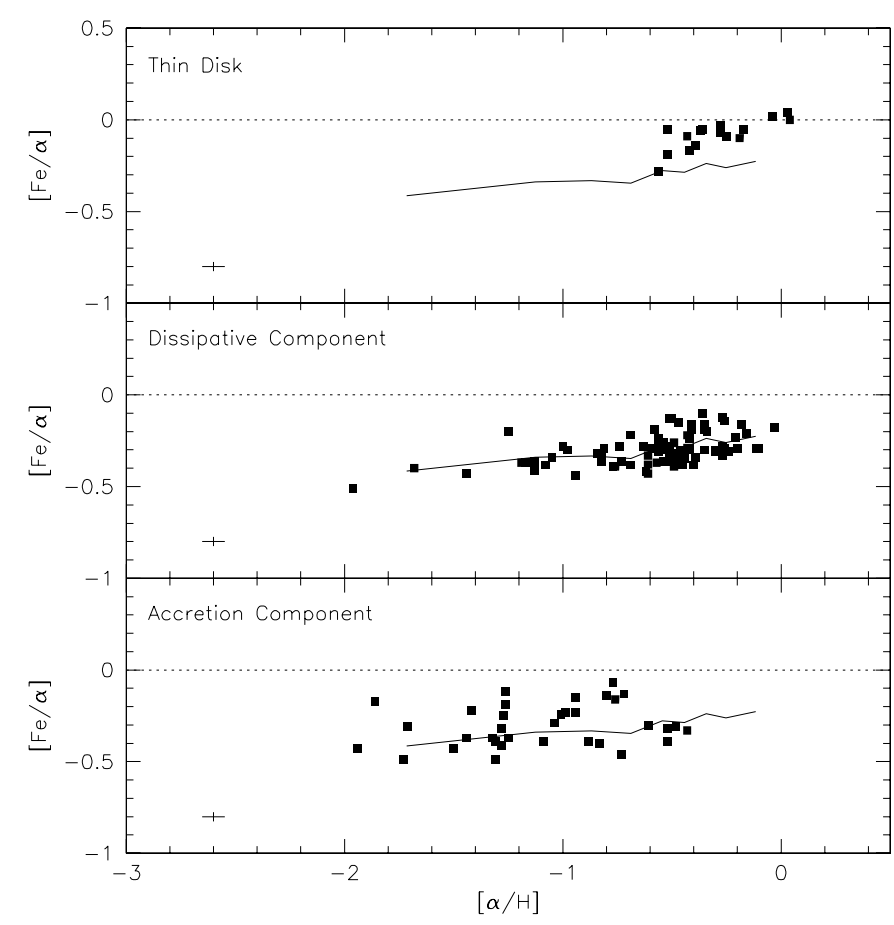

Fig. 2. Run of the abundance ratio of $\mathrm{Fe}$ to the average of the $\alpha$-elements vs. $[\alpha / \mathrm{H}]$. Upper panel: data for the Thin Disk stars; middle panel: data for Dissipative Component stars; lower panel: data for Accretion Component stars. The mean line through various metallicity bins for Dissipative component is overimposed in each plot. The typical error bar is shown in the lower left corner.

both $[\mathrm{Si} / \mathrm{Ca}]$ and $[\mathrm{Ca} / \mathrm{Ti}]$. Taking into account the errors in the $[\mathrm{Ca} / \mathrm{Fe}]$ ratio, there is still room for some real star-to-star scatter in these ratios, of about $0.063,0.055,0.026$ and $0.051 \mathrm{dex}$ for the $[\mathrm{O} / \mathrm{Ca}],[\mathrm{Mg} / \mathrm{Ca}],[\mathrm{Si} / \mathrm{Ca}]$ and $[\mathrm{Ti} / \mathrm{Ca}]$ ratios. While it is well possible that these residual scatter are due to (underestimated) observational errors, we note that again, there is a trend with atomic number that may be suggestive of a real nucleosynthetic origin. However, the conclusions of this paper will not depend on these considerations.

In the remaining part of this paper we will neglect the differences existing between individual $\alpha$-elements (hereinafter not considering $\mathrm{O}$ ), and consider their average abundance. Figure 2 displays the run of the abundance ratio between $\mathrm{Fe}$ and the average of the $\alpha$-elements with the average abundance of the $\alpha$-elements. Stars belonging to the different components are shown in the three panels of this figure. Figure 3 displays similar plots, this time for the abundance ratio between the average of the $\alpha$-elements and Fe with respect to the Fe abundance.

Table 1 lists the average abundances of the $[\mathrm{Fe} / \alpha]$ ratio in different metallicity bins for the dissipative component. There is a clear trend for increasing $[\mathrm{Fe} / \alpha]$ ratios with increasing metallicity: the linear correlation coefficient is $r^{2}=0.257$ over 85 stars, which is significant at a very high confidence level. The following best fit line seems to reproduce data very accurately:

$[\mathrm{Fe} / \alpha]=(0.113 \pm 0.021)[\alpha / \mathrm{H}]-(0.229 \pm 0.071)$

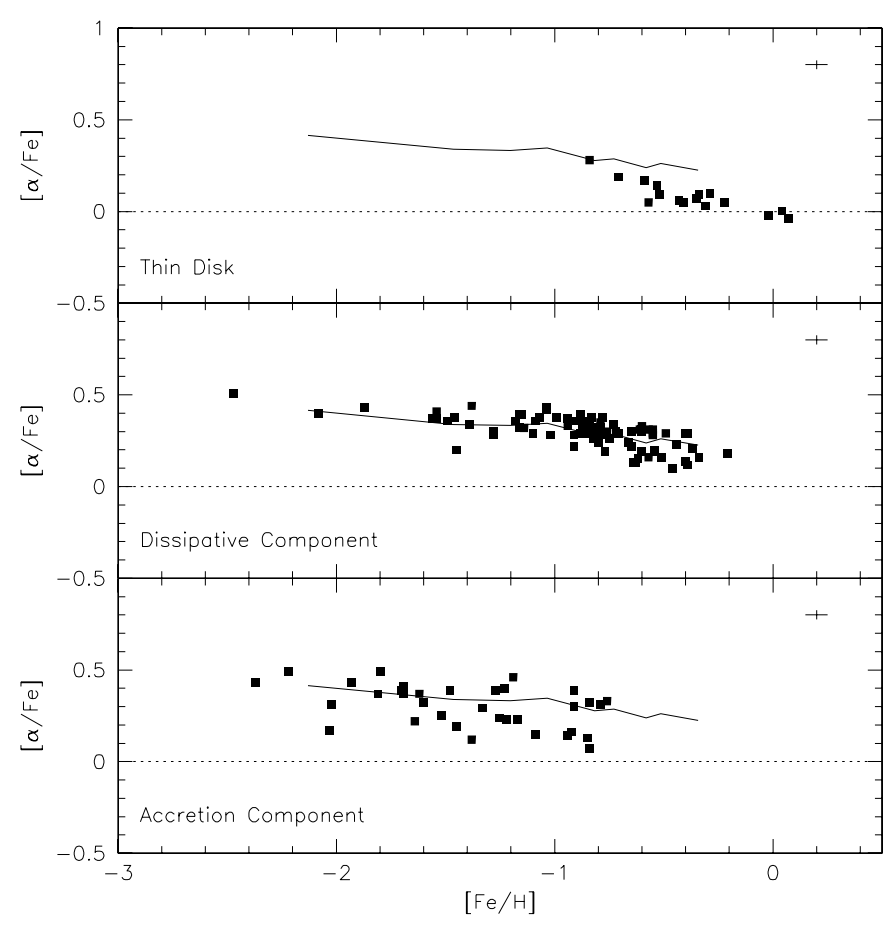

Fig. 3. Run of the abundance ratio of the average of the $\alpha$-elements to $\mathrm{Fe}$ vs. $[\mathrm{Fe} / \mathrm{H}]$. Upper panel: data for the Thin Disk stars; middle panel: data for Dissipative Component stars; lower panel: data for the Accretion Component stars. The mean line through various metallicity bins for Dissipative component is overimposed in each plot. The typical error bar is shown in the lower left corner.

Table 1. Average $[\mathrm{Fe} / \alpha]$ values for the dissipative component in various metallicity bins.

\begin{tabular}{crccc}
\hline \hline$[\alpha / \mathrm{H}]$ & $\begin{array}{r}\text { No. } \\
\text { bin }(\mathrm{dex})\end{array}$ & $\begin{array}{c}\langle\alpha / \mathrm{H}]\rangle \\
\text { Stars }\end{array}$ & $\begin{array}{c}{[\mathrm{Fe} / \alpha]} \\
(\mathrm{dex})\end{array}$ & $\begin{array}{c}\mathrm{rms} \\
(\mathrm{dex})\end{array}$ \\
\hline--1.3 & 4 & -1.715 & $-0.415 \pm 0.040$ & 0.079 \\
$-1.3--1.0$ & 8 & -1.125 & $-0.339 \pm 0.024$ & 0.067 \\
$-1.0--0.8$ & 7 & -0.869 & $-0.333 \pm 0.020$ & 0.053 \\
$-0.8--0.6$ & 12 & -0.688 & $-0.346 \pm 0.019$ & 0.065 \\
$-0.6--0.5$ & 15 & -0.544 & $-0.278 \pm 0.016$ & 0.066 \\
$-0.5--0.4$ & 16 & -0.443 & $-0.286 \pm 0.020$ & 0.078 \\
$-0.4--0.3$ & 8 & -0.342 & $-0.238 \pm 0.030$ & 0.086 \\
$-0.3--0.2$ & 10 & -0.253 & $-0.261 \pm 0.023$ & 0.074 \\
$-0.2-$ & 5 & -0.116 & $-0.226 \pm 0.027$ & 0.061 \\
\hline
\end{tabular}

where the error on the constant term represents the rms scatter along the line. This scatter is about three times the internal error (0.026 dex).

Part of this scatter is probably still due to analysis errors; however, we may use it to set lower limits to the mass of clouds undergoing independent chemical evolution during the formation of this component of the Milky Way. Following the same approach described in Carretta et al. (2002), we will assume for this exercise that the scatter around an average $[\mathrm{Fe} / \alpha]$ ratio is due to the stochastic effect of a sampling of the initial mass function by a discrete number of SN progenitors. Using the same precepts of Carretta et al., we then obtain that 
typically about $\geq 100 \mathrm{SNe}$ contributed to the enrichment of each cloud. The exact value of this lower limit depends on the uncertain dependence of yields on the stellar initial mass. On turn, the value of the Fe abundance may be used to constrain the dilution factor of the ejecta of the $\mathrm{SNe}$, and from the number of $\mathrm{SNe}$ and the yields we may then get an estimate for the mass of the whole cloud, that is of $\geq 10^{5} M_{\odot}$. This value is not much different from what Carretta et al. found for much more metal-poor stars, where the rms scatter but also the dilution factor are larger. As noticed by the referee of Carretta et al. (Bruce Carney), this value for the mass of the clouds is of the same order of magnitude of the Jeans mass at that epoch, and also of the same order of the mass of globular clusters. This may be perhaps considered then as a typical mass for clouds having an independent chemical evolution over a broad range of initial conditions in our Galaxy.

We overplotted the line representing the average values of the $[\mathrm{Fe} / \alpha]$ ratios for the dissipative component also on the two other panels of Fig. 2. Thin disk stars (shown in the upper panel) have a much larger average value of the $[\mathrm{Fe} / \alpha]$ ratio at a given metallicity; the mean residual with respect to the best fit line of Eq. (2) is $0.176 \pm 0.014 \mathrm{dex}$, with an rms of only $0.059 \mathrm{dex}$, only slightly larger then the internal error. This large difference in the $[\mathrm{Fe} / \alpha]$ ratios is simply confirmation of the results obtained by Gratton et al. $(1996,2000)$ and Fuhrmann (1998) for O and Mg. On the other side, stars belonging to the accretion component show a much larger scatter than that observed for stars of the dissipative component (the rms is of 0.108 dex over 41 star). An F-test shows that the difference between the scatter around the mean line for the dissipative collapse and the accretion components is significant at $99.9 \%$ level of confidence, while a Kolmogorov-Smirnov test indicate that the two distributions are different at $95.0 \%$ level of confidence. Also the average $[\mathrm{Fe} / \alpha]$ ratios of the two populations are different, in the sense that the $[\mathrm{Fe} / \alpha]$ values of the accretion component at a given metallicity are larger by $0.043 \pm 0.017 \mathrm{dex}$; this is significant at $97.6 \%$ probability level (Student's $t$ test). This result confirm an earlier finding (from a much more limited sample) by Nissen \& Schuster (1997).

Finally, before interpreting the observed slopes in the $[\mathrm{Fe} / \alpha]$ ratio as real, we should warn the reader that we performed an LTE analysis using 1-d plane parallel model atmospheres. 3-D and non-LTE effects are expected to depend on $[\mathrm{Fe} / \mathrm{H}]$, and may affect abundances of $\mathrm{Fe}$ and $\alpha$-elements in different ways. Hence, a closer non-LTE/3-D study should be undertaken before the important conclusion about type Ia $\mathrm{SNe}$ affecting the chemical evolution of the dissipative component can be considered definitive.

\section{Correlation with kinematics and biases}

Correlating our abundances with kinematics helps to understand several important features of our sample of metal-poor stars. In Fig. 4 we displayed the run of the metallicity (from Fe) with various kinematical parameters: Perigalactic distance $R_{\min }$ (upper left panel); Apogalactic distance $R_{\max }$ (lower left panel); Galactic orbit eccentricity $e$ (upper right panel); Rotational velocity around the Galactic center $v_{\text {rot }}$ (lower right panel).
Different symbols are used for stars belonging to the various populations considered throughout this paper. Similar plots, but with (average) metallicity from the $\alpha$-elements are shown in Fig. 5; while in Fig. 6 we plotted the kinematical quantities against the abundance ratio between $\mathrm{Fe}$ and the $\alpha$-elements.

Before opening a discussion on the results shown in these figures, we must warn the readers about the biases that affect our results. There are mainly three biases:

1. Our sample is drawn from the Hipparcos catalogue. This is complete only down to approximately $V \sim 7$; fainter stars (the vast majority of those included in the present sample) were obtained by combining lists proposed by several groups. These included a rather large number of high proper motion stars, so that there is a strong kinematical bias in our sample, favoring objects on highly eccentric orbits, with low rotational velocities around the galactic center, and with either large apogalactic or small perigalactic distances;

2. Among the stars in the Hipparcos catalogue, we essentially selected metal-poor stars, that is stars known to have $[\mathrm{Fe} / \mathrm{H}]<-0.8$ (although a few stars more metal rich than this limit are actually present in the sample). The analysis of Carretta et al. (2000) shows that our sample is nearly complete insofar stars with $[\mathrm{Fe} / \mathrm{H}]<-1.5$ and accurate parallaxes are considered, but it is far from being complete for more metal-rich stars. However there is a clear bias against metal-rich stars;

3. Finally our sample only includes stars with accurate parallaxes; this limits the volume sampled to a small region around the Sun, with a radius of the order of $100 \mathrm{pc}$, the actual value depending also on the absolute magnitude of the stars. This biases our results against stars in the bulge, and also against objects of the outer halo on orbits of low eccentricities.

It is very difficult to properly quantify these biases. However their impact on the results should be carefully considered in the following discussion. Our results cannot be used to draw correlations between metallicity and orbit parameters, a basic data that is much properly presented elsewhere (see e.g. Sandage \& Fouts 1987; Norris 1994). Here, we will concentrate on a comparison between distribution of abundance ratios among different populations, under the assumption that they are less affected by the above mentioned selection effects.

We note that in the case of the Dissipational Collapse Component the rotational velocity is clearly correlated with the overall metallicity: the linear correlation coefficients are $r^{2}=$ 0.101 and 0.161 if $[\alpha / \mathrm{H}]$ and $[\mathrm{Fe} / \mathrm{H}]$ are used respectively (over 85 stars, that is 83 degrees of freedom). Both these values are significant at more than $99 \%$ level of confidence. However, as mentioned above these correlations are at least in part artifacts of the selection biases present in our sample. We think that the (closer) correlation existing between the rotational velocity and the $[\mathrm{Fe} / \alpha]$ ratio is much more interesting. In this case, the linear correlation coefficient is $r^{2}=0.369$, over the same number of stars. This correlation is significant at a very high level of confidence; as a matter of fact, rotational velocity can be used 

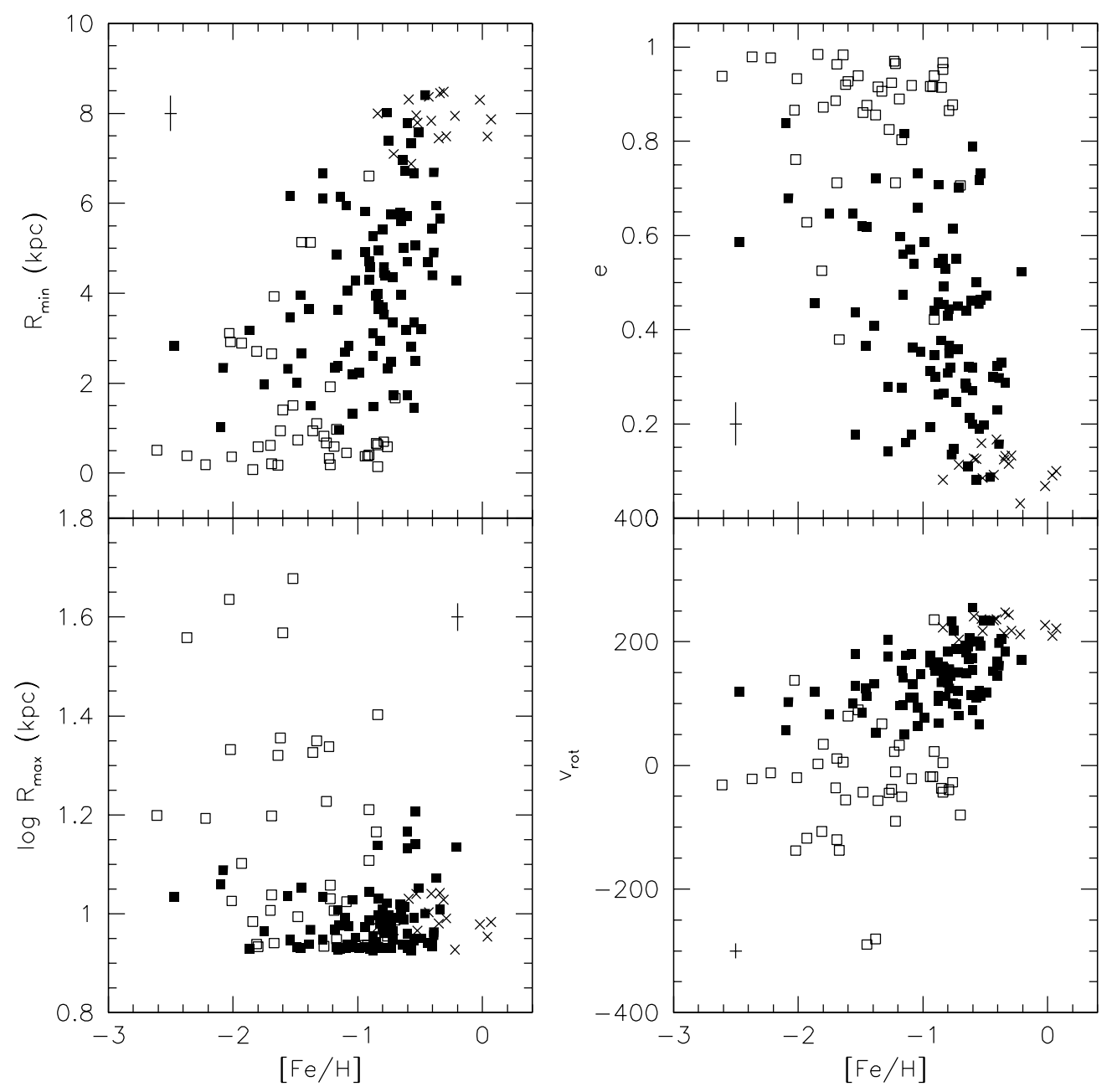

Fig. 4. Run of the metallicity (from Fe) with various kinematical parameters: Perigalactic distance $R_{\min }$ (upper left panel); Apogalactic distance $R_{\max }$ (lower left panel); Galactic orbit eccentricity $e$ (upper right panel); Rotational velocity around the Galactic center $v_{\text {rot }}$ (lower right panel). Filled squares are data for Dissipative Component stars; open squares those for the Accretion Component stars; crosses are data for Thin Disk stars. Typical error bars are shown in each panel.

to predict the value of the $[\mathrm{Fe} / \alpha]$ ratio with an accuracy better than that obtained using overall metallicity: the relation is:

$[\mathrm{Fe} / \alpha]=(0.00109 \pm 0.00016) V_{\text {rot }}-(0.454 \pm 0.065)$.

The fact that the $[\mathrm{Fe} / \alpha]$ ratio is better correlated with the rotational velocity than the overall metallicity is very interesting, because we expect that the effects of selection biases are in this case only indirect; that is, they should affect this correlation only through their impact on metallicity and rotational velocity.

\section{Discussion and conclusion}

Our large sample of metal-poor stars with accurate abundance analysis and kinematics allows a discussion of some of the chemical properties of the components related to the dissipative collapse (rotating component) and to accretion (either counter-rotating or not-rotating). When examining the plots of Figs. $4-6$, we should remind that $\alpha$-elements are mainly produced by core collapse SNe. They then follow closely the star formation history of the Galaxy. On the other side, since a significant fraction of $\mathrm{Fe}$ is produced on much longer time scales by thermonuclear $\mathrm{SNe}$, its production is somewhat delayed with respect to that of the $\alpha$-elements. Hence we expect that a $\mathrm{Fe} / \alpha$ ratio increasing with the time is a signature of a lower star formation rate.

The first feature we notice in our results is that even if both the dissipative collapse and accretion components show a large overlap in metallicity, they show distinct chemical properties. In fact, while in both the cases there is a significant deficiency of Fe with respect to the $\alpha$-elements, at a given metallicity the Fe deficiency is more pronounced, and the scatter is smaller, among stars belonging to the dissipational collapse component, in agreement with earlier results by other authors (Nissen \& Schuster 1997). As far as the dissipational component is concerned, the Fe deficiency outlined above is a signature of a star formation rate higher than that of the accretion component. Moreover the smaller scatter noticed above (Figs. 4-6) indicates that they formed from a more homogeneous interstellar medium that the accretion component, may be well mixed over regions typically as massive as $10^{5} M_{\odot}$. Finally for the dissipational component we derive a close correlation between chemical composition and kinematics, as expected for 

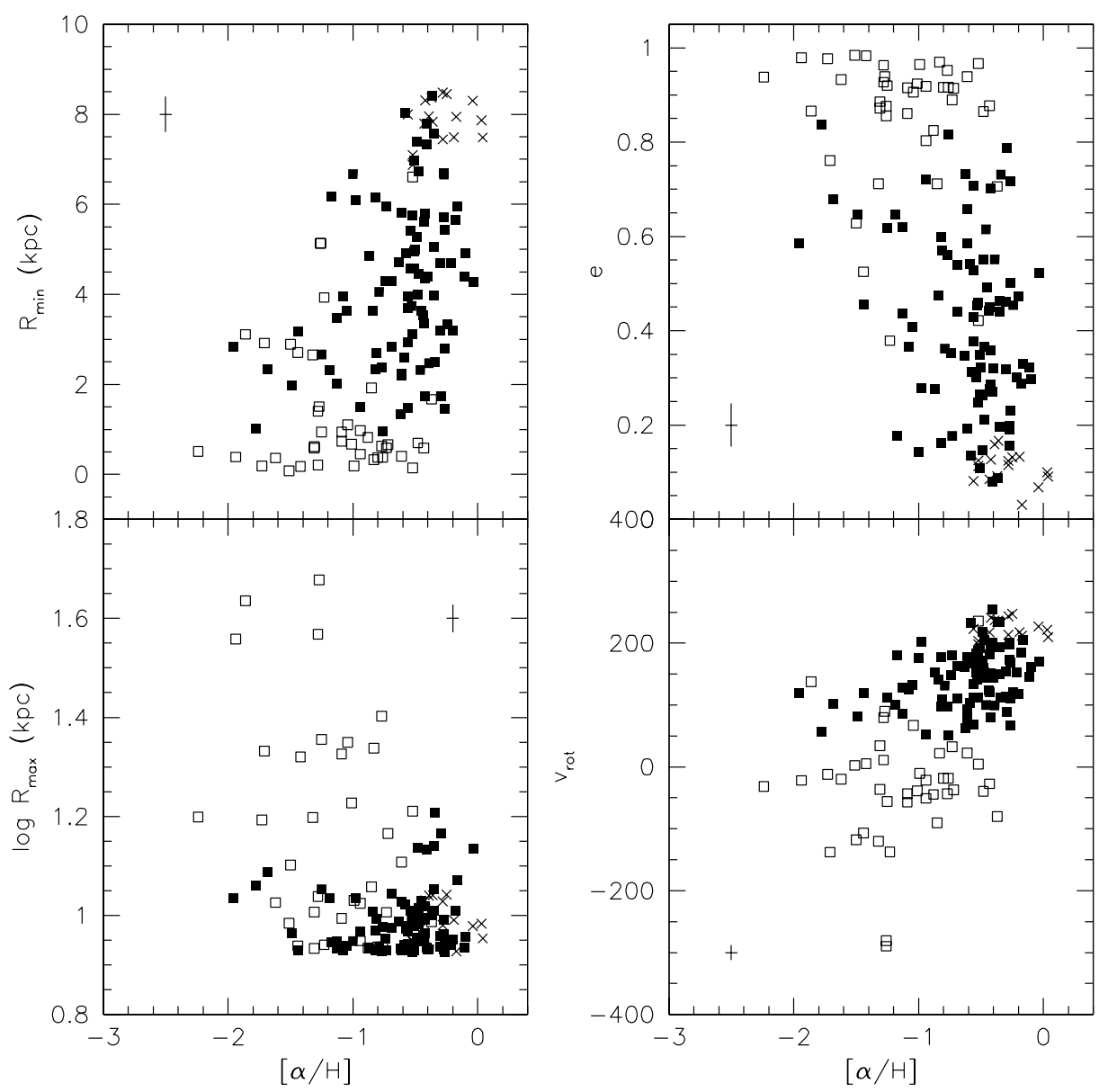

Fig. 5. Run of the metallicity (from $\alpha$-elements) with various kinematical parameters: Perigalactic distance $R_{\min }$ (upper left panel); Apogalactic distance $R_{\max }$ (lower left panel); Galactic orbit eccentricity $e$ (upper right panel); Rotational velocity around the Galactic center $v_{\text {rot }}$ (lower right panel). Filled squares are data for Dissipative Component stars; open squares those for the Accretion Component stars; crosses are data for Thin Disk stars. Typical error bars are shown in each panel.

a continuous metal enrichment during the dissipative collapse phase of the Galaxy. However, while star formation occurred quite vigorously, it still left time enough for a raise of the $[\mathrm{Fe} / \alpha]$ ratio from a value of $[\mathrm{Fe} / \alpha] \sim-0.40$ at $[\alpha / \mathrm{H}] \sim-1.5$, to a value of $[\mathrm{Fe} / \alpha] \sim-0.23$ at $[\alpha / \mathrm{H}] \sim 0$, where the evolution of this component interrupts. This trend is very clear, but needs to be confirmed by a 3-D/non-LTE study. As found by other authors (Gratton et al. 1996, 2000; Fuhrmann 1998; Reddy et al. 2003), this component is distinct from the thin disk, more rich in Fe, although shows an overlap in $[\alpha / \mathrm{H}]$. The raise of the $[\mathrm{Fe} / \alpha]$ ratio during formation of the dissipative collapse component suggests that this lasted long enough to allow about $1 / 3$ of the Fe produced by thermonuclear SNe to be deposited in the interstellar medium (again, with the caveat that this result might be due to 3 -D/non-LTE effects not considered in the present analysis). While the time scale for the evolution of the progenitors of these $\mathrm{SNe}$ is still not well defined (see the review in Nomoto et al. 1999), this result suggests that the collapse phase was perhaps not very short.

On the other side, the accretion component exhibits a much larger scatter in the $[\mathrm{Fe} / \alpha]$ ratio: this is essentially due to the presence of a significant population of Fe-rich stars, that made up nearly a third of the sample. We prefer the notation Fe-rich stars rather than the usual one, $\alpha$-poor, because it is more suggestive of the origin of the anomaly: these stars are in fact quite metal-rich, so that an interpretation of their anomalies as due to random fluctuations of primordial $\mathrm{SNe}$ is not very likely (it would require that these stars formed in small clouds with a typical mass of $3 \times 10^{4} M_{\odot}$ ). Moreover within this framework we do not expect a significant difference between the average $[\mathrm{Fe} / \alpha]$ ratio of the accretion and dissipative collapse populations, at variance with what we find here. It seems more plausible that these stars formed in an environment with lower star formation rate and a chemical evolution quite independent of that of the Milky Way, similar to that found in the dwarf spheroidal galaxies, whose chemical composition has been recently studied by Shetrone et al. (2003) and Tolstoy et al. (2003). They tend to have higher $\mathrm{Fe} / \alpha$ than stars in the dissipative component. Thus these stars should exhibit a large scatter in their ages.

As discussed in Sect. 4, selection biases can affect previous suggestions. Nevertheless we wish to call the attention of the reader to the last panel in Fig. 6, which shows the trend of the $[\mathrm{Fe} / \alpha]$ ratio with rotational velocity. As discussed above, the $[\mathrm{Fe} / \alpha]$ ratio is most likely an alias of time. While there is a clear bias in this diagram in the sense that stars with low 

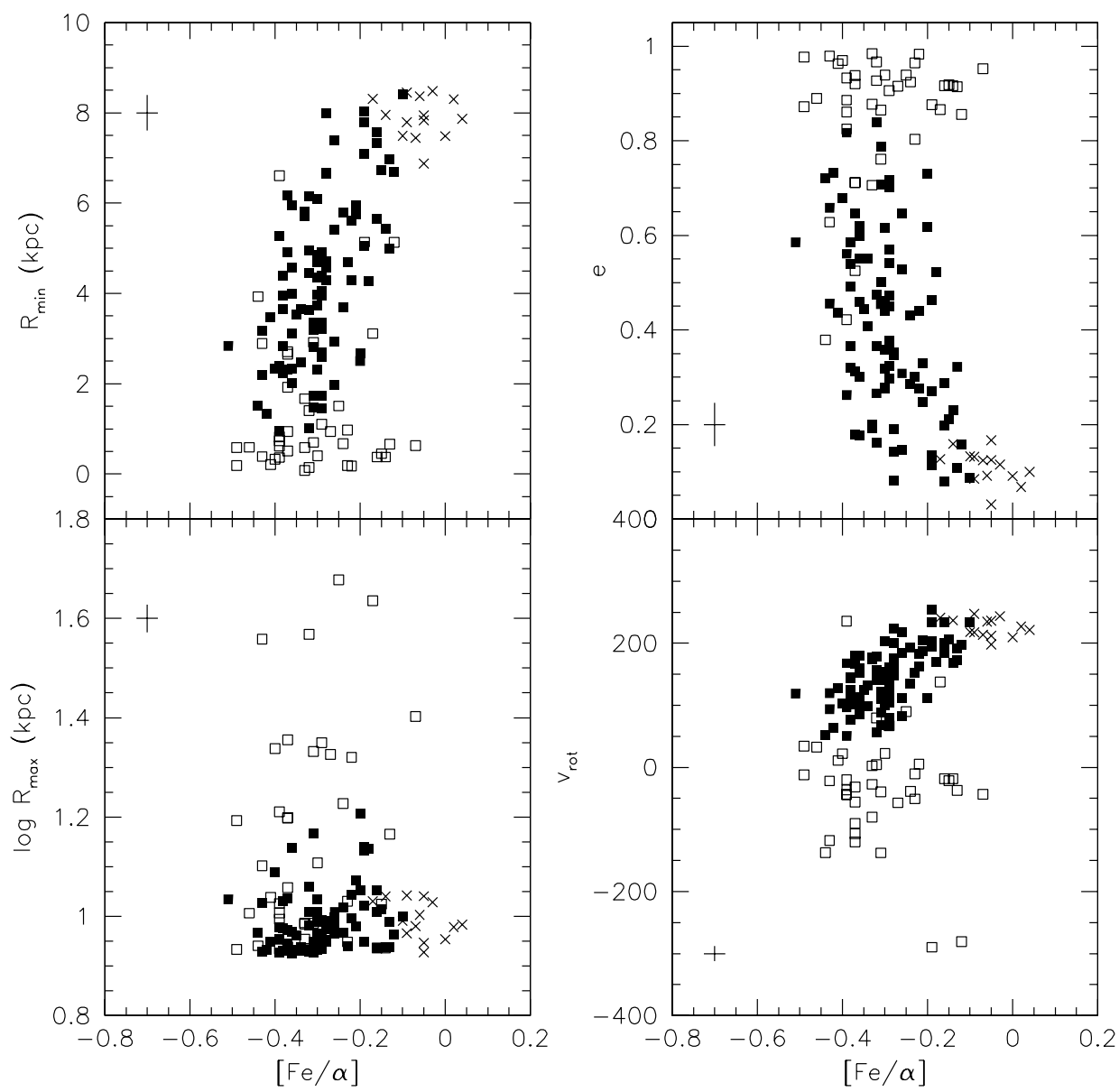

Fig. 6. Run of the abundance ratio between $\mathrm{Fe}$ and $\alpha$-elements with various kinematical parameters: Perigalactic distance $R_{\min }$ (upper left panel); Apogalactic distance $R_{\max }$ (lower left panel); Galactic orbit eccentricity $e$ (upper right panel); Rotational velocity around the Galactic center $v_{\text {rot }}$ (lower right panel). Filled squares are data for Dissipative Component stars; open squares those for the Accretion Component stars; crosses are data for Thin Disk stars. The typical error bars are shown in each panel as plus symbols.

rotational velocity are under-represented, there is no obvious bias at a given rotational velocity. Thus it is interesting to compare the distribution of the values of the $[\mathrm{Fe} / \alpha]$ ratio for stars belonging to the accretion component with that for stars with moderate rotational velocities $\left(40<V_{\text {rot }}<150 \mathrm{~km} \mathrm{~s}^{-1}\right)$. While in the first group roughly half of the stars have $[\mathrm{Fe} / \alpha]>-0.3$ (and so are probably significantly younger), stars as rich in $\mathrm{Fe}$ are very rare in the second group. The lack of slowly corotating stars with high $\mathrm{Fe} / \alpha$ abundance ratios in the solar neighborhood is expected from dynamical models of disk formation. However a significant population of slowly counterrotating Fe-rich stars points toward a long lifetime of their protogalactic clouds, in particular longer than that of the dissipational component. Moreover the possibility that accretion component stars belong to the bulge and that their kinematics properties have been changed by violent or two-body relaxation processes, is not appealing in view of their low metal abundance $([\mathrm{Fe} / \mathrm{H}]<-1)$.

Finally, we wish to point out that perigalactic distances correlate with the $\mathrm{Fe} / \alpha$ abundance ratios much better than the apogalactic distances. At first sight, this result is quite surprising, since we would expect that the apogalactic distance is a better signature of the region of formation of the star and that accretion component stars should form mainly in the outer halo of the Galaxy. However, it should be noticed that the sample here considered is drawn from the solar neighborhood, a region clearly distinct from the outer halo. In order to observe in the solar neighborhood stars formed in the outer halo, highly eccentric orbits are requested; if such an orbit passes very close to the galactic bulge, it becomes chaotic, losing most of the information about its initial conditions (i.e. apogalactic distances).

Acknowledgements. This research has made use of the SIMBAD data base, operated at CDS, Strasbourg, France; it was funded by COFIN 2001028897 by Ministero Università e Ricerca Scientifica, Italy. We thank the referee for his helpful comments.

\section{References}

Abadi, G. M., Navarro, J. F., Steinmetz, M., \& Eke, V. R. 2002, preprint [astro-ph0211331]

Alonso, A., Arribas, S., \& Martinez-Roger, C. 1996, A\&AS, 117, 227

Barklem, P. S., Piskunov, N., \& O'Mara, B. J. 2000, A\&AS, 142, 467

Carretta, E., Gratton, R. G., Clementini, G., \& Fusi Pecci, F. 2000, ApJ, 533, 215

Carretta, E., Gratton, R. G., Cohen, J. G., Beers, T. C., \& Christlieb, N. 2002, AJ, 124, 481 
Chiappini, C., Matteucci, F., \& Gratton, R. G. 1997, ApJ, 477, 765

Eggen, O. J., Lynden-Bell, D., \& Sandage, A. R. 1962, ApJ, 136, 748

Freeman, K., \& Bland-Hawthorne, J. S. 2002, ARA\&A, 40, 487

Fuhrmann, K. 1998, A\&A, 338, 161

Fulbright, J. P. 2000, AJ, 120, 1841

Gilmore, G., Wyse, R. F. G., \& Kujiken, C. 1989, ARA\&A, 27, 555

Gratton, R. G., Carretta, E., Matteucci, F., \& Sneden, C. 1996, in Formation of the Galactic Halo... Inside and Out, ed. H. Morrison, \& A. Sarajedini, ASP Conf. Ser., 92, 307

Gratton, R. G., Carretta, E., Matteucci, F., \& Sneden, C. 2000, A\&A, 358,671

Gratton, R. G., Carretta, E., Claudi, R., Lucatello, S., \& Barbieri, M. 2003, A\&A, 404, 187 (Paper I)

Governato, F., Mayer, L., Wadsley, et al. 2002, preprint [astro-ph0207044]

Ibata, R. A., Gilmore, G., \& Irwin, M. J. 1995, MNRAS, 277, 781

Kay, S. T., Pearce, F. R., Frenck, C. S., \& Jenkins, A. 2002, MNRAS, 330, 113

Kurucz, R. L. 1994, CD-ROM 19

Larson, R. B. 1974, MNRAS, 166, 585

Larson, R. B. 1976, MNRAS, 176, 31

Nissen, P. E., \& Schuster, W. J. 1997, A\&A, 326, 751
Nissen, P. E., Primas, F., Asplund, M., \& Lambert, D. L. 2002, A\&A, 390, 235

Nomoto, K., Umeda, H., Hachisu, I., et al. 1999, in Type Ia Supernovae: Theory and Cosmology, ed. J. C. Niemeyer, \& J. W. Truran (Cambridge: Cambridge Univ. Press), Chap. 6

Norris, J. E. 1994, ApJ, 431, 645

Perryman, M. A. C., Lindegren, L., Kovalevsky, J., et al. 1997, A\&A, 323, L49

Prochaska, J., Naumov, S. O., Carney, B. W., McWilliam, A., \& Wolfe, A. M. 2000, AJ, 120, 2513

Reddy, B. E., Tomkin, J., Lambert, D. L., \& Allende Prieto, C. 2003, MNRAS, in press [astro-ph/0211551]

Samland, M., \& Gerhard, O. E. 2003, A\&A, 399, 961

Sandage, A. R., \& Fouts, G. 1987, AJ, 93, 592

Searle, L., \& Zinn, R. 1978, ApJ, 225, 357

Shetrone, M., Venn, K. A., Tolstoy, E., Primas, F., Hill, V., \& Kaufer, A. 2003, AJ, 125, 684

Silk, J., \& Bouwens, R. 2001, New Astron. Rev., 45, 337

Steinmetz, M. 2001, in Astrophysical Supercomputing Using Particles, IAU Symp. 208, 1, ed. J. Makino, \& P. Hut

Tolstoy, E., Venn, K. A., Shetrone, M., et al. 2003, AJ, 125, 707

Weinberg, D. H., Hernquist, L., \& Katz, N. 2002, ApJ, 571, 15 